\title{
Phylogenetic relationships of Pleurotus species according to the sequence and secondary structure of the mitochondrial small-subunit rRNA V4, V6 and V9 domains
}

\author{
Patrice Gonzalez and Jacques Labarère
}

Laboratory of Molecular Genetics and Breeding of Cultivated Mushrooms, INRA-University Victor Segalen Bordeaux 2, BP 81, 33883 Villenave d'Ornon Cédex, France
Author for correspondence: Jacques Labarère. Tel: +33 5568431 69. Fax: +335568431 79. e-mail: labarere@bordeaux.inra.fr

\begin{abstract}
A comparative study of the V4, V6 and V9 domains of the mitochondrial smallsubunit (SSU) rRNA was conducted to evaluate the use of these sequences to investigate phylogenetic relatedness within the genus Pleurotus. The PCR products encompassing these regions from $\mathbf{4 8}$ isolates belonging to 16 Pleurotus species were sequenced and compared. From this comparison, the length and sequence of the three domains were found to be constant within a species. Significant inter-species variations due to insertion/deletion events were found, in most cases occurring in regions not directly involved in the maintainance of the standard SSU rRNA secondary structure. Phylogenetic analysis based upon these mitochondrial sequences was in agreement with relationships previously established by morphological descriptions and with previous studies based upon the nuclear genome or isozymes; moreover such analysis resolved some ambiguities in earlier analyses. It was confirmed that $P$. ostreatus and $P$. florida represent a single species, as well as $P$. pulmonarius and $P$. sajor-caju. The phylogenetic analysis also made it possible to assess the relative positions of $P$. rattenburyi, $P$. lampas, $P$. sapidus, $P$. colombinus and $P$. eryngii. The results clearly showed that sequences of the V4, V6 and V9 domains of the mitochondrial SSU rRNA could provide good markers for use in the taxonomy and phylogeny of species of Basidiomycota. Because of their nucleotide conservation, the major advantage of these species-specific markers was the possibility to study only one isolate from each species to determine phylogenetic relatedness.
\end{abstract}

Keywords: Basidiomycota, mitochondrial SSU rRNA, Pleurotus, taxonomy, phylogeny

\section{INTRODUCTION}

Most taxomonic and phylogenetic studies of Basidiomycota have been based on the analysis of morphological characters. In the last 10 years, relationships among species in several genera of Basidiomycota have often been established by RFLP studies of the nuclear genome, and by amplification of nuclear sequences by PCR. RFLP analysis allowed separation of species belonging to the genera Laccaria (Gardes et al., 1990), Armillaria (Smith \& Anderson, 1989) and Sclerotinia

Abbreviations: ITS, internal transcribed spacer; SSU, small subunit.

The GenBank accession numbers for the sequences reported in this paper are given in Methods.
(Khon et al., 1988). PCR investigations have mainly focused on nucleotide sequences of the internal transcribed spacer (ITS) located between the nuclear rDNA $18 \mathrm{~S}$ and $28 \mathrm{~S}$ subunit genes, and made it possible to determine the relationships between fungal species from the genus Ganoderma (Molcalvo et al., 1995).

Little use has hitherto been made of extrachromosomal genetic information constituted by the mitochondrial DNA in Basidiomycota. The mitochondrial genomes usually contain structural genes for subunits involved in the respiratory chain, and genes for components of the mitochondrial protein synthesis machinery, i.e. a complete set of tRNAs, the large and the small subunits of the rRNA (LSU and SSU rRNAs) (for a review see Barroso \& Labarère, 1995). Mitochondrial 
DNA has been used in several studies to determine phylogenetic relationships of the coleopteran genus Hegeter (Juan et al., 1996), the dipteran Drosophila buzzatiis (Spicer, 1995), the Ascomycota Allomyces macrogynus (Paquin et al., 1995) and the protist Euglena gracilis (Teissier et al., 1997). All these phylogenetic analyses based upon mitochondrial genes (cox1, cyt $b$ and SSU rRNA) have given congruent results when compared to results obtained from nuclear genomes.

Recently the sequence and secondary structure of the mitochondrial SSU rRNA from the Basidiomycota Agrocybe aegerita was determined (Gonzalez et al., 1997). Analysis indicated that in this SSU rRNA, three domains, V4, V6 and V9, possessed unusually long nucleotide extensions. Comparisons of these sequences with partial $5^{\prime}$ sequences overlapping the V4 domain of 80 other Basidiomycota species, available in databases (Bruns \& Szaro, 1992; Hibbet \& Donoghue, 1995), showed that length variations of the V4 domain seemed to be species-specific. We previously found that sequences of the three domains are species-specific in the genus Agrocybe and could be used to determine phylogenetic relationships between species (Gonzalez \& Labarère, 1998). These results suggested that such mitochondrial sequences could constitute a new approach in the taxonomy and phylogeny of microorganisms. Because of the invariability of these sequences within a species, the major advantage of this approach is the need for only one isolate from each species to determine the phylogenetic relationship. Unfortunately, no other phylogenetic study was available in the literature on the genus Agrocybe to infer confidence in the use of these sequences in phylogeny of Basidiomycota.

In this study, we investigated the lengths and sequences of the V4, V6 and V9 domains among the genus Pleurotus (order Poriales). Pleurotus species are widespread mushrooms that usually grow on wood; they include cultivated species of important economic value such as $P$. ostreatus, $P$. pulmonarius, $P$. cornucopiae and P. cystidiosus. Moreover, the genus Pleurotus has been more thoroughly investigated than the genus Agrocybe, and numerous phylogenetic studies based upon the analysis of molecular markers are available; they have been based upon the comparison of isozyme patterns (Zervakis et al., 1994), the analysis of the nuclear genome by RFLP (Iraçabal et al., 1995) and sequence comparisons of the ITS (Vilgalys \& Sun, 1994). More recently, intercompatibility studies have been carried out for some species of Pleurotus (Zervakis \& Balis, 1996). So it appeared particularly interesting to compare our analysis based upon mitochondrial sequences with previous phylogenetic analysis based upon nuclear markers. Moreover, all these previous studies have failed to resolve some phylogenetic ambiguities, such as the taxonomic position of $P$. sapidus, $P$. eryngii, $P$. colombinus and $P$. flabellatus. In addition, most previous studies classify the species $P$. pulmonarius and $P$. sajor-caju in the same species complex (Zervakis et al., 1994; Iraçabal et al., 1995), and P. florida has been described as a probable subspecies of $P$. ostreatus (Guzman et al., 1994). In this context, it was interesting to investigate whether the use of mitochondrial sequences could resolve some of these ambiguities.

In this work we used specific primers flanking the V4, V6 and V9 domains of the mitochondrial SSU rRNA (Gonzalez \& Labarère, 1998) to amplify mitochondrial DNAs from 48 isolates from 16 Pleurotus species (Table $1)$; isolates were chosen according to their different geographical origin. PCR products were purified and sequenced. Alignment of the resulting sequences allowed us to deduce nucleotide sequence variations and the putative evolution of each mitochondrial domain. We determined the secondary structures of each domain and used these to regroup species within subgroups. We established a consensus tree based upon the concatemerized sequences of the V4, V6 and V9 domains using the neighbour-joining, the parsimony and the UPGMA methods. We then compared the resulting trees to previously reported phylogenetic analysis of Pleurotus.

\section{METHODS}

Pleurotus strains and culture medium. All the Pleurotus strains used were dikaryotic (Table 1). They were grown in Petri dishes $(90 \mathrm{~mm})$ containing $30 \mathrm{ml}$ potato dextrose agar (PDA) medium (39 $\mathrm{g} \mathrm{l}^{-1}$; Sigma), in the dark at $26^{\circ} \mathrm{C}$ (FPN 70 incubator, Friga-Bohn) for $8-14 \mathrm{~d}$. The geographical origins of the strains used are reported in Table 1.

Genomic DNA purification. Total DNA was extracted from vegetative mycelia with the $N$-cetyl-N,N,N-trimethylammonium bromide (CTAB) method adapted for a small quantity of mycelium (Barroso et al., 1995). Mycelium (around $0 \cdot 2 \mathrm{~g}$ ) was collected with a scalpel from an $8 \mathrm{~d}$ culture, frozen in liquid nitrogen and crushed in a mortar. The crushed mycelium was resuspended in $0.7 \mathrm{ml}$ extraction buffer (100 mM Tris/ $\mathrm{HCl} \mathrm{pH} 8,2 \%$, w/v, CTAB, $20 \mathrm{mM}$ EDTA, $1.4 \mathrm{M} \mathrm{NaCl}, 2 \%, \mathrm{v} / \mathrm{v}, \beta$-mercaptoethanol) and incubated for $20 \mathrm{~min}$ at $56^{\circ} \mathrm{C}$. Then $0.7 \mathrm{ml}$ chloroform/isoamyl alchohol $(24: 1, \mathrm{v} / \mathrm{v})$ was added and the two phases were mixed to obtain an emulsion. After centrifugation $(9000 \mathrm{~g}, 15 \mathrm{~min}$, $20{ }^{\circ} \mathrm{C}$ ), the aqueous phase was removed and again subjected to a second extraction with $0.7 \mathrm{ml}$ chloroform/isoamyl alcohol, as described above. Nucleic acids were precipitated by $0.7 \mathrm{ml}$ precipitation buffer $(50 \mathrm{mM}$ Tris $/ \mathrm{HCl} \mathrm{pH} \mathrm{8,1 \% ,} \mathrm{w/v,} \mathrm{CTAB,}$ $10 \mathrm{mM}$ EDTA, $1 \%, \mathrm{v} / \mathrm{v}, \beta$-mercaptoethanol) for $30 \mathrm{~min}$ at room temperature. The precipitate was recovered by centrifugation $\left(9000 \mathrm{~g}, 15 \mathrm{~min}, 20^{\circ} \mathrm{C}\right)$, dried, resuspended in $0.5 \mathrm{ml}$ $1 \mathrm{M} \mathrm{NaCl}$ and incubated for $20 \mathrm{~min}$ at $56^{\circ} \mathrm{C}$. Nucleic acids were then precipitated at room temperature by adding 2 vols absolute ethanol. After centrifugation $(11000 \mathrm{~g}, 15 \mathrm{~min}$, $20{ }^{\circ} \mathrm{C}$ ), the pellet was washed three times with $1 \mathrm{ml} 70 \%$ (v/v) ethanol to completely eliminate the excess of CTAB. The pellet was dried and then resuspended in sterile distilled water. Nucleic acids were used directly for PCR amplifications or stored at $4^{\circ} \mathrm{C}$.

PCR amplification. Amplifications were performed using the previously described primer pairs V4U/V4R, V6U/V6R and V9U/V9R (Gonzalez \& Labarère, 1998) to amplify the regions containing, respectively, the variable domains V4, V6 and V9 of the mitochondrial SSU rDNA. These primer pairs were complementary to regions flanking the V4, V6 and V9 
Table 1. Length of the variable domains V4, V6 and V9 of the mitochondrial SSU rDNA from 49 strains belonging to 16 species of Pleurotus

\begin{tabular}{|c|c|c|c|c|c|c|c|c|c|c|c|}
\hline \multirow{3}{*}{$\begin{array}{l}\text { Pleurotus groups, } \\
\text { species and } \\
\text { strains* }\end{array}$} & \multirow[t]{3}{*}{ Origin $†$} & \multicolumn{4}{|c|}{ V4 domain } & \multicolumn{3}{|c|}{ V6 domain } & \multicolumn{3}{|c|}{ V9 domain } \\
\hline & & \multirow[t]{2}{*}{$\begin{array}{c}\text { Length } \\
\text { (nt) } \ddagger\end{array}$} & \multicolumn{3}{|c|}{$\begin{array}{l}\text { Secondary } \\
\text { structures } \mathbb{S}\end{array}$} & \multirow[t]{2}{*}{$\begin{array}{c}\text { Length } \\
(\mathrm{nt}) \neq\end{array}$} & \multicolumn{2}{|c|}{$\begin{array}{l}\text { Secondary } \\
\text { structures } \mathbb{S}\end{array}$} & \multirow[t]{2}{*}{$\begin{array}{c}\text { Length } \\
(\mathbf{n t}) \ddagger\end{array}$} & \multicolumn{2}{|c|}{$\begin{array}{l}\text { Secondary } \\
\text { structures } \$\end{array}$} \\
\hline & & & P23-2 & $\mathrm{P} 23-1$ & P23-3 & & P37-1 & P37-2 & & P49-2 & P49-1 \\
\hline \multicolumn{12}{|l|}{ GROUP A } \\
\hline \multicolumn{12}{|l|}{ A1 } \\
\hline \multicolumn{12}{|l|}{ P. citrinopileatus } \\
\hline SC 971213 & MUCL 28684 & 77 & + & - & - & 266 & ++ & - & 236 & - & + \\
\hline \multicolumn{12}{|l|}{ P. euosmus } \\
\hline SC 971222 & CBS 307.29 & 71 & + & - & - & 264 & ++ & - & 223 & - & + \\
\hline \multicolumn{12}{|l|}{$\mathrm{A} 2$} \\
\hline \multicolumn{12}{|l|}{ P. cornucopiae } \\
\hline SC 960601 & $\operatorname{Japan}^{b}$ & 77 & + & - & - & 82 & + & - & 225 & - & + \\
\hline SC 960602 & Japan $^{b}$ & 77 & + & - & - & 82 & + & - & 225 & - & + \\
\hline A3 & & & & & & & & & & & \\
\hline P. cystidiosus & & & & & & & & & & & \\
\hline SC 910413 & CBS 615.80 & 69 & + & - & - & 75 & + & - & 208 & - & $\|$ \\
\hline SC 960902 & Thailand $^{b}$ & 69 & + & - & - & 75 & + & - & 208 & - & $\|$ \\
\hline SC 910415 & CBS 298.35 & 69 & + & - & - & 75 & + & - & 208 & - & $\|$ \\
\hline A4 & & & & & & & & & & & \\
\hline P. dryinus & & & & & & & & & & & \\
\hline SC 971232 & CBS 278.90 & 69 & + & - & - & 195 & + & + & 256 & - & + \\
\hline SC 971228 & CBS 804.85 & 69 & + & - & - & 195 & + & + & 256 & - & + \\
\hline A5 & & & & & & & & & & & \\
\hline P. smithii & & & & & & & & & & & \\
\hline SC 971230 & CBS 703.94 & 183 & + & - & + & 91 & + & - & 230 & - & + \\
\hline GROUP B & & & & & & & & & & & \\
\hline P. colombinus & & & & & & & & & & & \\
\hline SC 971214 & MUCL 31682 & 107 & + & + & - & 83 & + & - & 261 & + & + \\
\hline P. eryngii & & & & & & & & & & & \\
\hline SM 81 & France (south-west) ${ }^{b}$ & 106 & + & + & - & 83 & + & - & 241 & + & + \\
\hline SM 831102 & France (south-east) ${ }^{b}$ & Id & + & + & - & Id & + & - & ld & + & + \\
\hline SM 851101 & France (north-west $)^{b}$ & Id & + & + & - & Id & + & - & Id & + & + \\
\hline SC 871024 & Spain (Encantad) $)^{b}$ & 106 & + & + & - & 83 & + & - & 241 & + & + \\
\hline SC 920510 & Spain $^{b}$ & Id & + & + & - & Id & + & - & Id & + & + \\
\hline SM 901004 & Greece $(\text { Oriestiada })^{b}$ & 106 & + & + & - & 83 & + & - & 241 & + & + \\
\hline SM 901017 & Greece $(\text { Lasithi) })^{b}$ & Id & + & + & - & Id & + & - & Id & + & + \\
\hline SM 901028 & Greece (LGAM P 66) ${ }^{a}$ & Id & + & + & - & Id & + & - & Id & + & + \\
\hline SM 901029 & Greece (LGAM P 63) & Id & + & + & - & Id & + & - & Id & + & + \\
\hline SC 910404 & CBS 100.82 & 106 & + & + & - & 83 & + & - & 241 & + & + \\
\hline SC 910426 & ATCC 36047 & Id & + & + & - & Id & + & - & Id & + & + \\
\hline P. flabellatus & & & & & & & & & & & \\
\hline SC 971209 & MUCL 31687 & 90 & + & + & - & 82 & + & - & 267 & + & + \\
\hline SC 971212 & MUCL 28912 & 90 & + & + & - & 82 & + & - & 267 & + & + \\
\hline P. ostreatus & & & & & & & & & & & \\
\hline SM 841101 & France (south-west) $)^{b}$ & 104 & + & + & - & 83 & + & - & 267 & + & + \\
\hline SM 910407 & CBS 291.47 & Id & + & + & - & Id & + & - & Id & + & + \\
\hline SM 960913 & Italy $^{b}$ & 104 & + & + & - & 83 & + & - & 267 & + & + \\
\hline Sm 851001 & Czechoslovakia $^{b}$ & 104 & + & + & - & 83 & + & - & 267 & + & + \\
\hline SM 901007 & Greece (Vardoussia) $)^{b}$ & Id & + & + & - & Id & + & - & Id & + & + \\
\hline SM 901031 & Greece (LGAM P 38) ${ }^{a}$ & 104 & + & + & - & 83 & + & - & 267 & + & + \\
\hline SM 40 & India $(\text { Kashmir })^{b}$ & Id & + & + & - & Id & + & - & Id & + & + \\
\hline SM 960907 & Thailand $^{b}$ & 104 & + & + & - & 83 & + & - & 267 & + & + \\
\hline
\end{tabular}


Table 1 (cont.)

\begin{tabular}{|c|c|c|c|c|c|c|c|c|c|c|c|}
\hline \multirow{3}{*}{$\begin{array}{l}\text { Pleurotus groups, } \\
\text { species and } \\
\text { strains* }\end{array}$} & \multirow[t]{3}{*}{ Origin $†$} & \multicolumn{4}{|c|}{ V4 domain } & \multicolumn{3}{|c|}{ V6 domain } & \multicolumn{3}{|c|}{ V9 domain } \\
\hline & & \multirow[t]{2}{*}{$\begin{array}{l}\text { Length } \\
\text { (nt) } \neq\end{array}$} & \multicolumn{3}{|c|}{$\begin{array}{l}\text { Secondary } \\
\text { structures } \mathbb{}\end{array}$} & \multirow[t]{2}{*}{$\begin{array}{c}\text { Length } \\
\text { (nt) } \neq\end{array}$} & \multicolumn{2}{|c|}{$\begin{array}{l}\text { Secondary } \\
\text { structures } \mathbb{S}\end{array}$} & \multirow[t]{2}{*}{$\begin{array}{c}\text { Length } \\
(\mathbf{n t}) \ddagger\end{array}$} & \multicolumn{2}{|c|}{$\begin{array}{l}\text { Secondary } \\
\text { structures } \mathbb{}\end{array}$} \\
\hline & & & P23-2 & P23-1 & P23-3 & & P37-1 & P37-2 & & P49-2 & P49-1 \\
\hline \multicolumn{12}{|l|}{ P. florida } \\
\hline SC 971203 & MUCL 31688 & 104 & + & + & - & 83 & + & - & 267 & + & + \\
\hline SC 971208 & MUCL 31686 & 104 & + & + & - & 83 & + & - & 267 & + & + \\
\hline \multicolumn{12}{|l|}{ P. pulmonarius } \\
\hline SM 920512 & Spain $^{b}$ & 90 & + & + & - & 78 & + & - & 233 & + & + \\
\hline SM 920523 & Hungary $^{b}$ & 90 & + & + & - & 78 & + & - & 233 & + & + \\
\hline SM 901001 & Greece (Vardoussia) $)^{b}$ & 90 & + & + & - & 78 & + & - & 233 & + & + \\
\hline SM 901012 & Greece (Vardoussia) $)^{b}$ & Id & + & + & - & Id & + & - & Id & + & + \\
\hline SM 901022 & Greece $(\text { Vardoussia })^{b}$ & Id & + & + & - & Id & + & - & Id & + & + \\
\hline SM 960606 & $\operatorname{Japan}^{b}$ & Id & + & + & - & Id & + & - & Id & + & + \\
\hline SM 960607 & $\operatorname{Japan}^{b}$ & Id & + & + & - & Id & + & - & Id & + & + \\
\hline SM 920542 & CBS 132.85 & 90 & + & + & - & 78 & + & - & 233 & + & + \\
\hline \multicolumn{12}{|l|}{ P. sajor-caju } \\
\hline SM 37 & Mauritius $^{a}$ & 90 & + & + & - & 78 & + & - & 233 & + & + \\
\hline SC 960905 & Thailand $^{b}$ & 90 & + & + & - & 78 & + & - & 233 & + & + \\
\hline SC 960906 & Thailand $^{b}$ & 90 & + & + & - & 78 & + & - & 233 & + & + \\
\hline SC 971205 & MUCL 28685 & Id & + & + & - & Id & + & - & Id & + & + \\
\hline \multicolumn{12}{|l|}{ P. sapidus } \\
\hline SC 971223 & CBS 195.92 & 102 & + & + & - & 84 & + & - & 262 & + & + \\
\hline \multicolumn{12}{|l|}{ GROUP C } \\
\hline \\
\hline \multicolumn{12}{|l|}{ P. lampas } \\
\hline SC 971226 & CBS 323.49 & 71 & + & - & - & 100 & + & - & 284 & + & + \\
\hline \multicolumn{12}{|l|}{$\mathrm{C} 2$} \\
\hline \multicolumn{12}{|l|}{ P. rattenburyi } \\
\hline SC 971224 & CBS 175.94 & 67 & 9 & - & - & 82 & + & - & 415 & - & + \\
\hline
\end{tabular}

* The strain numbers correspond to the nomenclature of the Laboratory of Molecular Genetics and Breeding of Cultivated Mushrooms. +CBS, Centraalbureau Voor Schimmelcultures, Baarn, The Netherlands; MUCL, Mycothèque de I'Université Catholique du Louvainla-Neuve, Belgium; ATCC, American Type Culture Collection, Manassas, VA, USA. Isolates previously used by Iraçabal et al. (1995) are marked ' $a$ '; isolates used only in this study are marked ' $b$ '.

$\ddagger$ Id, length determined by agarose gel electrophoresis.

$\mathbb{S}+$, Presence; - , absence; ++, long P37-1 helix; \|, truncated P49-1 helix; 9, truncated P23-2 helix.

domains; they were determined by comparison with partial sequences available in databases and from the $A$. aegerita mitochondrial SSU rRNA sequence (Gonzalez et al., 1997). PCR reactions contained $50 \mathrm{mM} \mathrm{KCl}, 10 \mathrm{mM}$ Tris $/ \mathrm{HCl} \mathrm{pH} \mathrm{9,}$ $0 \cdot 1 \%$ Triton X-100, $0 \cdot 2 \mathrm{mM}$ of each dNTP, $2 \cdot 5 \mathrm{mM} \mathrm{MgCl}{ }_{2}$ and $3-5 \mu \mathrm{l}$ purified DNA in a final volume of $25 \mu \mathrm{l}$. Forty cycles were performed in a PTC 100 thermal cycler (MJ Research) as follows. DNA was denatured for $30 \mathrm{~s}$ at $95^{\circ} \mathrm{C}$, annealing of primers was obtained at a temperature corresponding to $T_{\mathrm{m}}-2{ }^{\circ} \mathrm{C}$ (i. e. 42,54 and $50{ }^{\circ} \mathrm{C}$ for amplification of the regions overlapping the V4, V6 and V9 domains, respectively) for $30 \mathrm{~s}$, and an elongation step was performed for $30 \mathrm{~s}$. PCR products were then analysed in a $1.5 \%$ agarose gel, or in a $5 \%$ polyacrylamide gel, and observed after ethidium bromide staining.
Purification and sequencing of PCR amplification products. Amplimers were purified using the Quiaquick PCR purification kit (QIAGEN), following the manufacturer's instructions to remove all the excess primer prior to sequencing reactions.

Sequencing of PCR products was performed on both strands using the ThermoSequenase sequencing kit (United States Biochemical) according to Sanger et al. (1977) with $[\alpha-$ ${ }^{33} \mathrm{P}$ ]ddNTPs $\left(55.5 \mathrm{TBq} \mathrm{mmol}^{-1}\right.$, Amersham). The primer pairs V4U/V4R, V6U/V6R and V9U/V9R were used to sequence PCR products of the variable domains V4, V6 and $\mathrm{V} 9$, respectively. The sequencing products were analysed in $6 \%$ polyacrylamide gels and revealed after exposure to Kodak $\mathrm{X}$-omat LS films. 


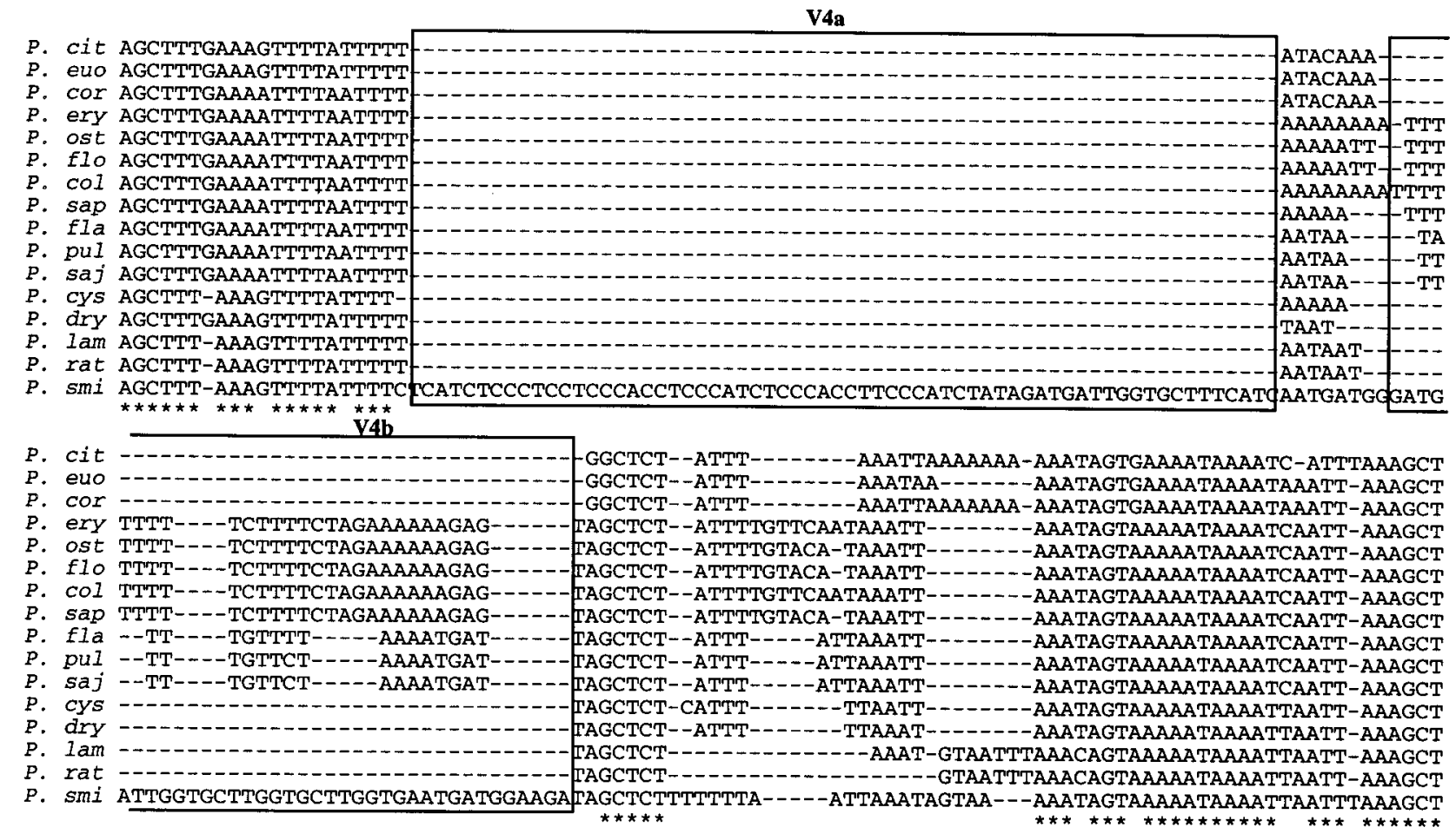

Fig. 1. Alignment of the V4 domain sequences of 16 species of the genus Pleurotus. Sequences strictly conserved among the 16 species are indicated by asterisks. The location and the putative size of insertion/deletion events are indicated by dashes. V4a and V4b boxes represent putative insertions/deletions events. $P$. cit, P. citrinopileatus SC $971213 ; P$. euo, P. euosmus SC 9712 22; P. cor, P. cornucopiae SC 9606 01; P. ery, P. eryngii SM 81; P. ost, P. ostreatus SM 841101 ; P. flo, P. florida SC 9712 03; P. col, P. colombinus SC 9712 14; P. sap, P. sapidus SC 9712 23; P. fla, P. flabellatus SC 971209 ; P. pul, P. pulmonarius SM 9205 12; P. saj, P. sajor-caju SM37; P. cys, P. cystidiosus SC $910413 ;$ P. dry, P. dryinus SC 9712 32; P. lam, P. lampas SC 9712 26; P. rat, P. rattenburyi SC 9712 24; P. smi, P. smithii SC 971230.

GenBank accession numbers. For the V4 domain: P. citrinopileatus, AF091891; P. colombinus, AF091892; P. cornucopiae, AF091893; P. cystidiosus, AF091894; P. dryinus, AF091895; P. eryngii, AF091896; P. euosmus, AF091897; P. flabellatus, AF091898; P. florida, AF 091899; P. lampas, AF091900; P. ostreatus, AF 091901; P. pulmonarius, AF091902; P. rattenburyi, AF091903; P. sajor-caju, AF091904; P. sapidus, AF091905; P. smithii, AF091906. For the V6 domain: P. citrinopileatus, AF091907; P. colombinus, AF091908; P. cornucopiae, AF091909; P. cystidiosus, AF091910; P. dryinus, AF091911; P. eryngii, AF091912; P. euosmus, AF091913; P. flabellatus, AF091914; P. florida, AF 091915; P. lampas, AF091916; P. ostreatus, AF 091917; P. pulmonarius, AF091918; P. rattenburyi, AF091919; P. sajor-caju, AF091920; P. sapidus, AF091921; P. smithii, AF091922. For the V9 domain: P. citrinopileatus, AF091923; P. colombinus, AF091924; P. cornucopiae, AF091925; P. cystidiosus, AF091926; P. dryinus, AF091927; P. eryngii, AF091928; P. enosmus, AF091929; P. flabellatus, AF091930; P. florida, AF 091931; P. lampas, AF091932; P. ostreatus, AF 091933; P. pulmonarius, AF091934; P. rattenburyi, AF091935; P. sajor-caju, AF091936; $P$. sapidus, AF091937; P. smithii, AF091938.

Phylogenetic analysis. Sequences were aligned with CLUSTAL w (version 1.7) software (Higgins \& Sharp, 1989). Consensus trees were constructed with the UPGMA, the neighbourjoining and the parsimony methods of the phylogeny inference package PHYLIP (version 3.5). To infer confidence of the branchpoints in the constructed tree, we performed a bootstrap analysis (1000 bootstrap replicates).

\section{RESULTS}

\section{PCR amplification and sequencing of the V4, V6 and V9 domain within 16 Pleurotus species}

We extracted total DNA from 48 isolates belonging to 16 Pleurotus species (Table 1), and amplified by PCR regions overlapping the V4, V6 and V9 domains of the mitochondrial SSU rRNA gene as previously described (Gonzalez \& Labarère, 1998). Agarose gel electrophoresis of the PCR products showed that a single amplimer was obtained for each domain; there were no significant intra-species length variations, whereas large inter-species size variations were observed.

For each species, we purified and sequenced PCR products from one to four isolates of different geographical origin, and precisely determined the lengths of the three domains (Table 1 ). The results confirmed preliminary analysis of PCR amplimer patterns obtained by agarose gel electrophoresis, i.e. all strains from the same species possessed exactly the same domain length, while there was great inter-species variation in size. The V4 domain ranged from $67 \mathrm{nt}(P$. rattenburyi) to $183 \mathrm{nt}$ (P. smithii), the V6 domain from 75 nt (P. cystidiosus) to $266 \mathrm{nt}$ ( $P$. citrinopileatus), and the V9 domain from $208 \mathrm{nt}$ ( $P$. cystidiosus) to $415 \mathrm{nt}$ ( $P$. rattenburyi). Some species possessed the same length for one domain: $90 \mathrm{nt}$ 

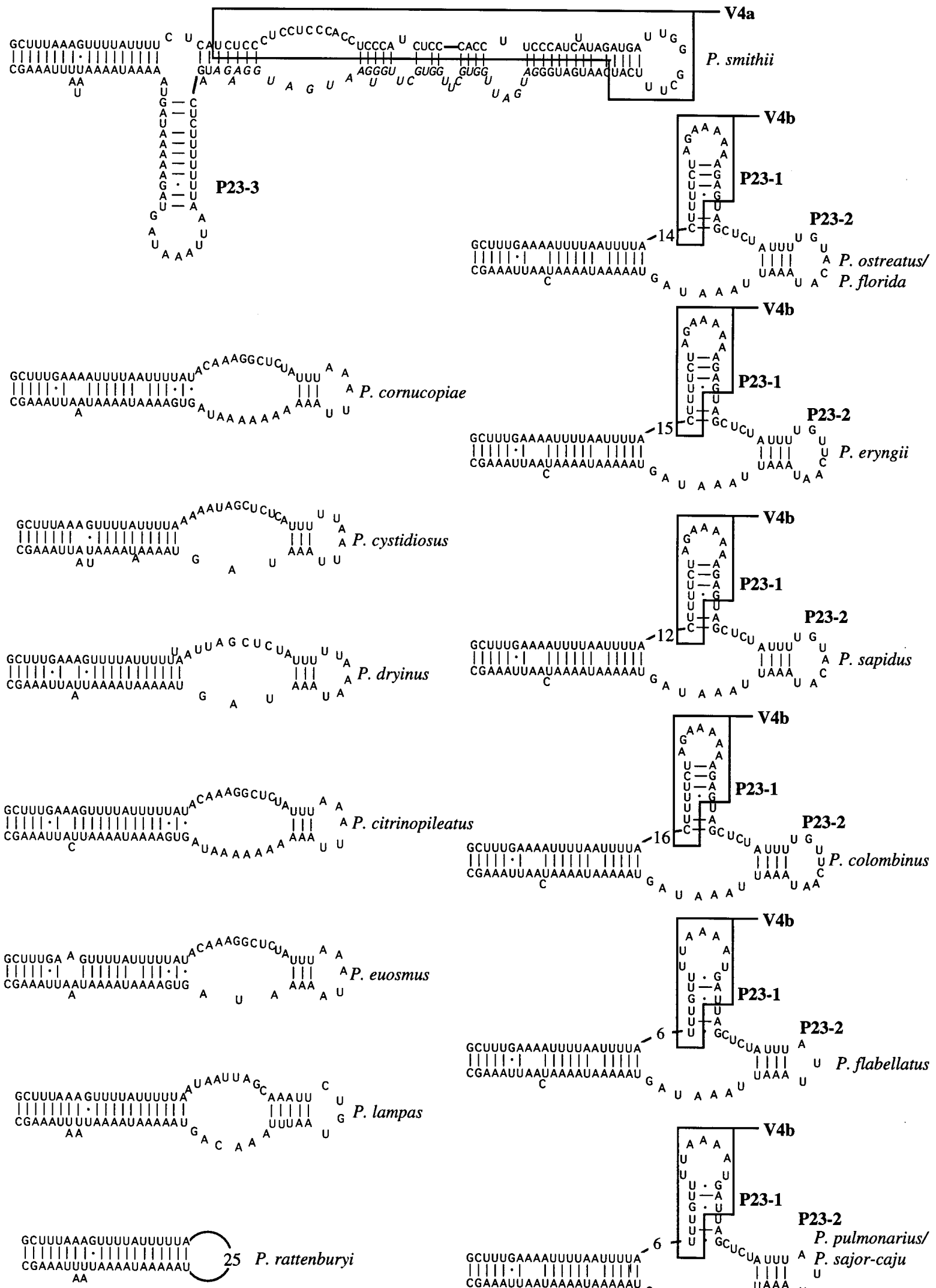

$P$. rattenburyi

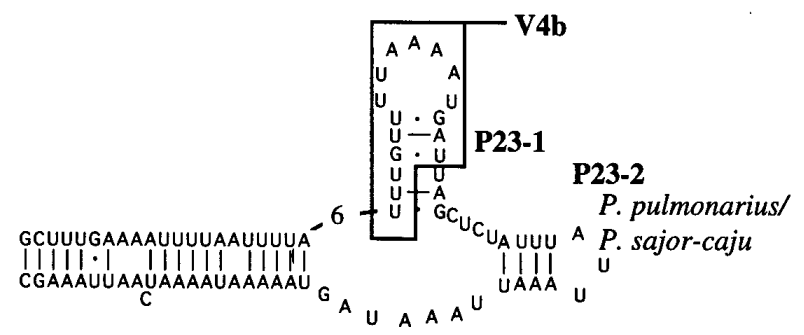

Fig. 2. Proposed secondary structure of the variable domain V4 of 16 species of the genus Pleurotus. The number of the additional helix is indicated. Nucleotides constituting the V4a and V4b motifs are framed. 
(P.pulmonarius, P. sajor-caju and P. flabellatus) or $77 \mathrm{nt}$ (P. citrinopileatus and $P$. cornucopiae) or $71 \mathrm{nt}(P$. lampas and $P$. euosmus) for the V4 domain; $82 \mathrm{nt}(P$. cornucopiae, P. flabellatus and $P$. rattenburyi) or $83 \mathrm{nt}$ (P. colombinus, P. florida and P. ostreatus) for the V6 domain; and $267 \mathrm{nt}$ (P. flabellatus, P. florida and P. ostreatus) for the V9 domain. Only two groups of species $-P$. pulmonarius ( 8 isolates) and P. sajor-caju (4 isolates) on the one hand, and P. florida (2 isolates) and P. ostreatus (8 isolates) on the other hand - shared the same V4, V6 and V9 lengths.

\section{Sequence comparisons of the V4, V6 and V9 domains within the genus Pleurotus}

Alignments of the nucleotide sequences showed that the sequences flanking the three variable domains were very similar among the 16 Pleurotus species studied (78$100 \%$ identity of either the V4, V6 or V9 flanking regions). In contrast, lower percentages of identity $(<70 \%)$ were observed when the V4, V6 and V9 sequences were compared.

For most of the species included in the study, several isolates were studied (2-11), and sequence comparisons showed that all isolates from the same species possessed identical sequences for the V4, V6 or V9 domains, respectively. Moreover, in the case of different species with identical nucleotide length for one of the three domains, these sequences appeared to be speciesspecific. Indeed, the occurrence of nucleotide changes allowed us to discriminate these species; for example, $P$. flabellatus ( 2 isolates) and $P$. pulmonarius (8 isolates), which both have a V4 domain of $90 \mathrm{nt}$, differed by two nucleotide base differences, while $P$. flabellatus (2 isolates) and $P$. ostreatus (8 isolates) (V9 $=267 \mathrm{nt}$ ) differed by 35 nucleotide changes. However, two groups of species $-P$. pulmonarius (8 isolates) and $P$. sajor-caju (4 isolates) on the one hand and P. ostreatus (8 isolates) and P. florida ( 2 isolates) on the other hand - shared exactly the same nucleotide sequence for each domain studied (Fig. 1).

Sequence alignment showed conserved nucleotide motifs within the genus. Three were located in the V4 domain (Fig. 1) : AAARTTTTAWTTT in the $5^{\prime}$ part, GCTCT in the central part, and AAAYAGTRAAAATAAAAT in the $3^{\prime}$ part. Another conserved motif was located in the $5^{\prime}$ part of the V6 domain (CATATYTA) and two in the V9 domain: AWTAWAACTT in the $5^{\prime}$ part, and CATTTRASTMA in the $3^{\prime}$ part.

For each domain the nucleotide motifs constituting the smallest sequence were present in the largest one, suggesting an evolution by sets of insertion/deletion events. For example, P. smithii, possessing the largest V4 domain (183 nt), differed from the other species mainly by the presence of the additional nucleotide motifs V4a and V4b. The V4a motif is absent in all other species studied, while the V4b motif is partially found in six species ( $P$. eryngi, P. ostreatus, $P$. colombinus, $P$. sapidus, P. flabellatus, P. pulmonarius) (Fig. 1).
We observed similar differences in the V6 and the V9 domains. Indeed, in the V6 domain, a TATAGC motif found in P. lampas was absent in all other species and two sequences of $129 \mathrm{nt}$ and $39 \mathrm{nt}$, found in P. citrinopileatus, $P$. dryinus and $P$. euosmus were absent in the other species. In the V9 domain a $10 \mathrm{nt}$ motif present in $P$. rattenburyi and $P$. lampas was absent in the remaining species and a $136 \mathrm{nt}$ motif found in $P$. rattenburyi was absent in all other species except $P$. flabellatus and $P$. citrinopileatus, in which a few residues from this sequence were present (15 nt and $6 \mathrm{nt}$, respectively).

\section{Comparison of the putative secondary structures of the V4, V6 and V9 domains within the genus Pleurotus}

With the aim of precisely locating where the insertion/ deletion events had occurred, the putative secondary structures of all domains for each species were determined. It should be noted that $P$. pulmonarius and $P$. sajor-caju on the one hand, and P. ostreatus and $P$. florida on the other hand, sharing the same sequences for the three domains, possessed the same secondary structures.

For the V4 domain, four groups of putative secondary structures were observed; they were characterized by the presence or absence of additional helices V4-P23-1 and V4-P23-3 and by the length of the major helix V4P23-2 (Fig. 2). In one group ( $P$. citrinopileatus, $P$. enosmus, $P$. cornucopiae, $P$. cystidiosus, $P$. dryinus, $P$. smithii, P. lampas), the V4 domain possessed a canonical topology (Neefs et al., 1993) without additional helices. Another group (P. colombinus, P. eryngii, P. flabellatus, P. ostreatus $/ P$. florida, P. pulmonarius $/ P$. sajor-caju, $P$. sapidus) was characterized by the presence of the additional helix V4-P23-1. Interestingly, the sequence of this helix was strictly conserved among the species $P$. colombinus, $P$. eryngii, $P$. ostreatus $/ P$. florida and $P$. sapidus on the one hand, and in P. flabellatus and P. pulmonarius/P. sajor-caju on the other hand. Another group (P. smithii) possessed the additional helix V4-P233 . The last group ( $P$. rattenburyi) possessed a truncated V4-P23-2 helix. From the comparison of these secondary structures, it appeared that sequence variations in the V4 domain were not randomly scattered; they were essentially due to (i) differences in the number of nucleotides in the loops, (ii) the presence of additional helices and (iii) the reduction of the V4-P23-2 helix.

Three types of secondary structures were found for the V6 domain (Fig. 3). In most species (P. cornucopiae, $P$. cystidiosus, P. smithii, P. colombinus, P. eryngii, $P$. flabellatus, P. ostreatus/P. florida, P. pulmonarius $/ P$. sajor-caju, $P$. sapidus, P. lampas, $P$. rattenburyi), this domain possessed the single and common V6-P37-1 helix. One variant group, including $P$. euosmus and $P$. citrinopileatus, possessed an unusually large V6-P37-1 with more than 120 base-paired nucleotides. The third 

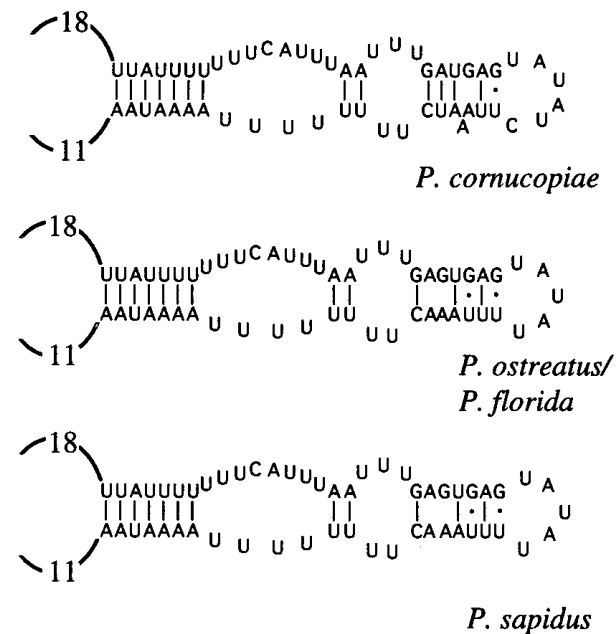

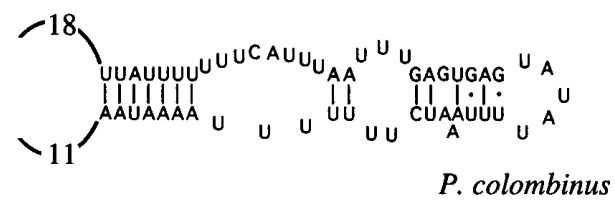

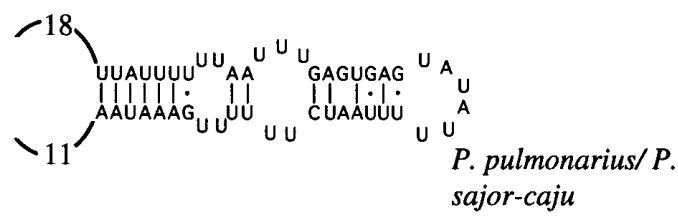

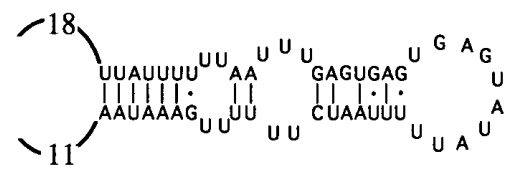

P. flabellatus
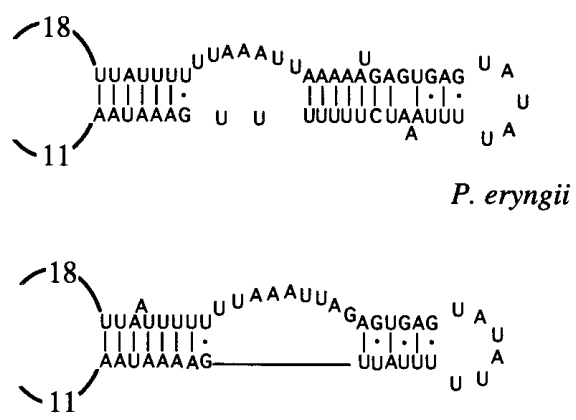

P. cystidiosus

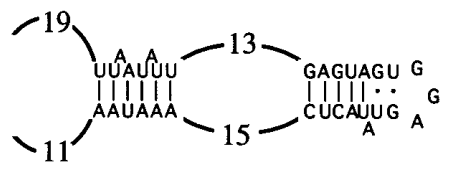

P. smithii
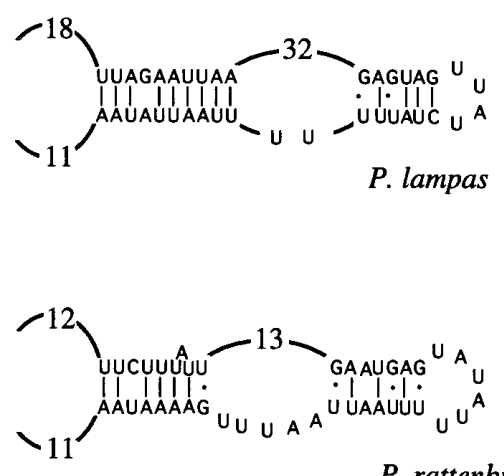

$P$. rattenburyi
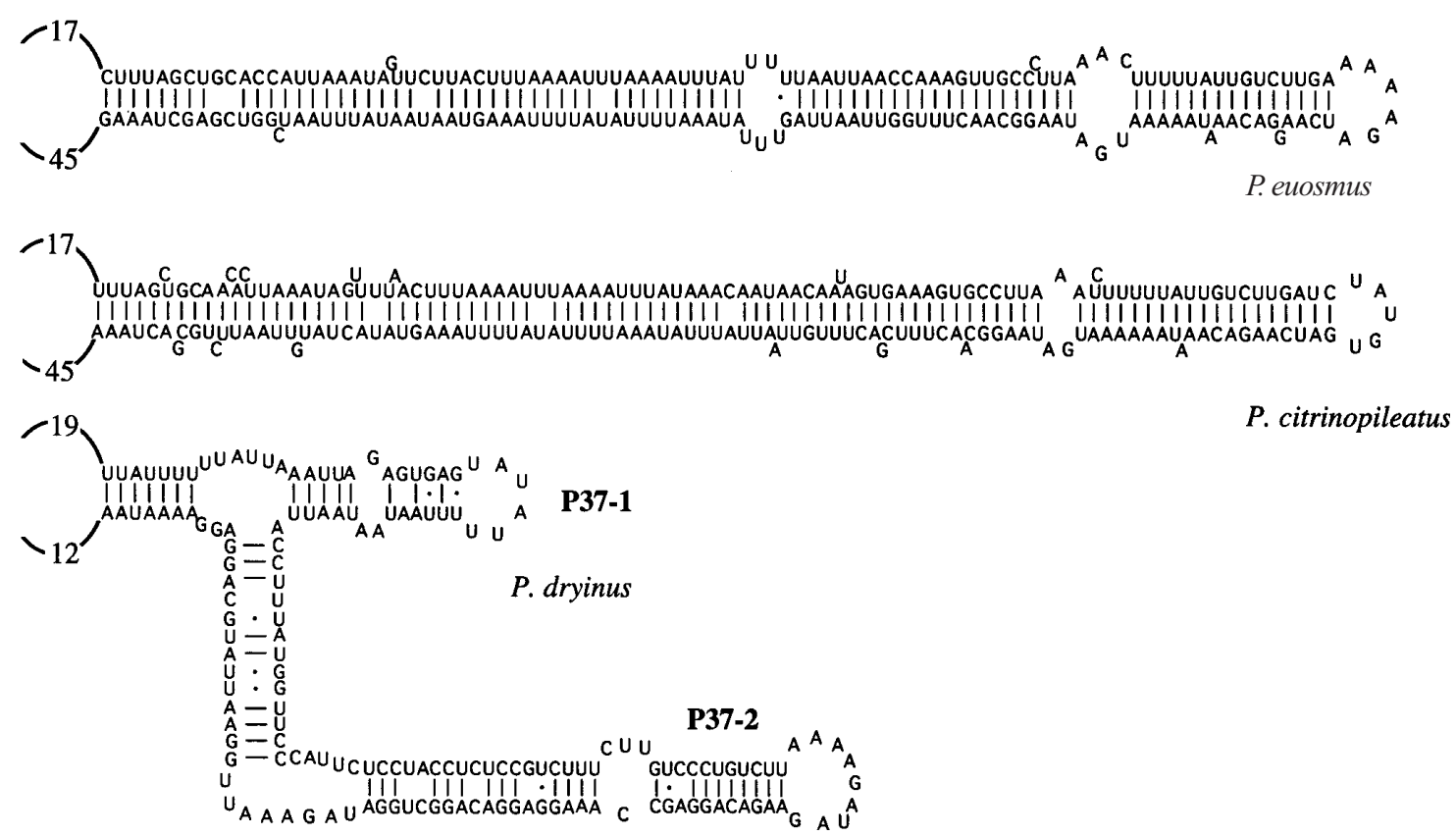

Fig. 3. Proposed secondary structure of the variable domain V 6 of 16 species from the genus Pleurotus. The number of the additional helix is indicated. 
group included only one species ( $P$. dryinus), which possessed the additional helix V6-P37-2. As in the V4 domain, length variations in the V6 domain were due to variations in the length of the V6-P37-1 helix and of the loops, and to the occurrence of the V6-P37-2 helix.

We observed three groups of secondary structures for the V9 domain (Fig. 4): one possessed a classical V9P49-1 helix (P. citrinopileatus, P. euosmus, P. cornucopiae, $P$. dryinus, $P$. smithii, $P$. rattenburyi), one was characterized by a truncated V9-P49-1 helix (P.cystidiosus), and one by an additional V9-P49-2 helix (P. colombinus, P. eryngii, P. flabellatus, P. ostreatus/ P. florida, P. pulmonarius $/ P$. sajor-caju, P. sapidus, P. lampas).

\section{Phylogenetic analysis of Pleurotus species based on the V4, V6 and V9 domain sequences}

The sequences of the three variable domains of each species were all joined together in the order V4-V6-V9 and used to construct consensus trees with the UPGMA, the neighbour-joining and the parsimony methods (PHYLIP package, version 3.5). Similar phylogenetic trees were obtained using the neighbour-joining and the parsimony methods, whereas minor changes were apparent in the consensus tree constructed with the UPGMA method. In the UPGMA tree, three groups (A, B, C) of species were clearly recovered (Fig. 5a). Group A was divided into two subgroups: one included $P$. citrinopileatus and P. euosmus, closely related to each other and associated with P. cornucopiae; the other included $P$. dryinus and P. smithii, strongly associated with $P$. cystidiosus. Group B also contained two subgroups related to $P$. ostreatus $/ P$. florida: one subgroup included $P$. pulmonarius $/ P$. sajor-caju, closely related to $P$. eryngii; the other subgroup included $P$. sapidus associated with $P$. colombinus and themselves related to $P$. flabellatus. Group $\mathrm{C}$ consisted of $P$. rattenburyi and $P$. lampas.

The major differences observed in the neighbourjoining/parsimony tree concerned the relationships among group $B$ species and the relative position of group C. In this tree (Fig. 5b) P. ostreatus/P. florida were more closely related to $P$. sapidus and $P$. colombinus, instead of being considered as the most divergent species of this group (Fig. 5a). Also, P. flabellatus appeared related to $P$. pulmonarius $/ P$. sajor-caju and P. eryngii. Moreover, group $B$ was not so clearly separated from the other species as in the UPGMA tree. Group C appeared to be inserted between groups A and B in this tree, while it was considered as the most divergent group in the UPGMA tree. However, it should be noted that in the neighbour-joining/parsimony tree, the divergent positions were less supported by the bootstrap analysis than those observed in the UPGMA tree. For example, the phylogenetic position of $P$. ostreatus $/ P$. florida possessed a bootstrap support of $67.5 \%$ in the UPGMA analysis and only $53-58 \%$ in the other analysis.

\section{DISCUSSION}

\section{Species-specificity of the V4, V6 and V9 sequences within the Pleurotus genus}

Previous studies have shown nucleotide conservation in the sequences of the V4, V6 and V9 domains within the genus Agrocybe (Gonzalez \& Labarère, 1998). Analysis of these sequences in the present study for several isolates of $P$. eryngii (11 isolates), P. ostreatus (8 isolates), $P$. pulmonarius (8 isolates) or P. sajor-caju (4 isolates) confirmed that sequences of the three domains are species-specific. Regarding the results obtained with about 90 strains in either Agrocybe or Pleurotus genera, the mitochondrial SSU rRNA V4, V6 and V9 domains do not seem to have evolved since speciation. This nucleotide conservation within a species might be correlated with the ability of these domain sequences to bind specific nucleus-encoded riboproteins involved in obtaining the efficient three-dimensional folding of the 30S subunit (Noller \& Lake, 1984; Stern et al., 1989; Mueller \& Brimacombe, 1997). In the light of our results, it could be hypothesized that these sequences are under strong physiological and/or molecular constraints that avoid significant sequence modifications within species. Thanks to their nucleotide conservation, these sequences constitute useful species-specific markers for taxonomy and phylogeny of Basidiomycota. Consequently, the major advantage of using these mitochondrial sequences is the need for only one isolate of each species to determine phylogenetic relationships. By comparison, in nuclear ITS sequences, which are to date the most investigated sequences in phylogenetic studies, numerous intra-species nucleotide variations were found. For example, 10 nucleotide variations have been described in the ITS1 sequences and four in the ITS2 sequences from two isolates (D383 and D1136) of $P$. cornucopiae (Vilgalys \& Sun, 1994). So, when using nuclear ITS sequences to determine phylogenetic relatedness it is necessary to study numerous isolates from the same species.

\section{Inter-species variations and secondary structure of the V4, V6 and V9 domains within the genus Pleurotus}

As previously described for the genus Agrocybe, the three domains show inter-species variations, mainly due to point mutations and to insertion/deletion of nucleotide motifs that strengthen the hypothesis of an evolution of mitochondrial SSU rRNA sequences by insertion/ deletion events.

Determination of the secondary structure of each domain showed that insertion/deletion events are not randomly scattered, but essentially located in loops not directly involved in obtaining the mitochondrial SSU rRNA secondary structure. Based upon the V4, V6 and V9 secondary structures, Pleurotus species are organized in eight subgroups (Table 1). It is interesting to note that these subgroups are in agreement with previous morphological studies (Singer, 1986). For example, subgroup 


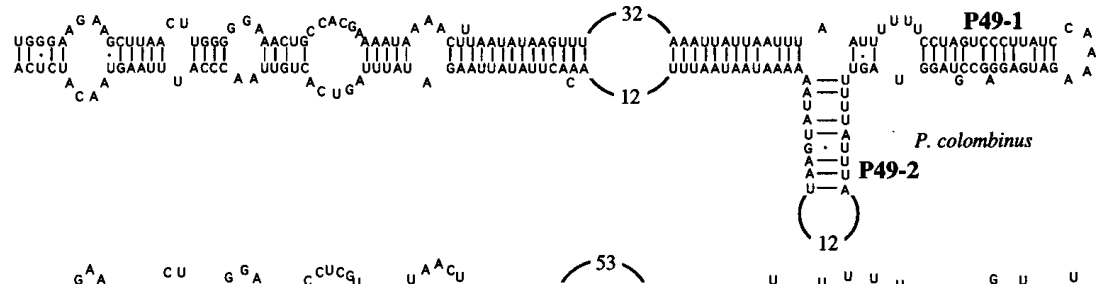

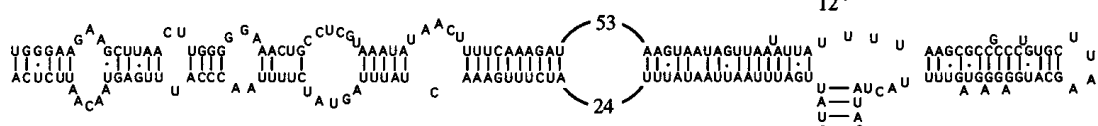

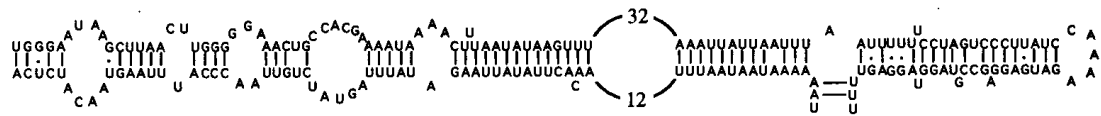

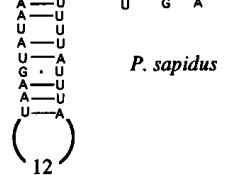

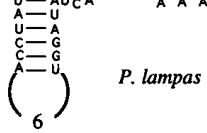

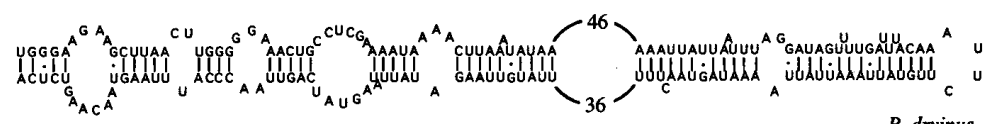

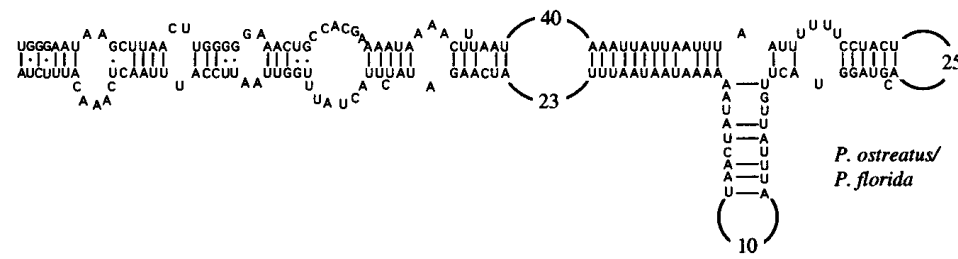

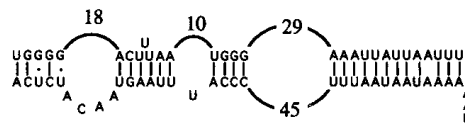

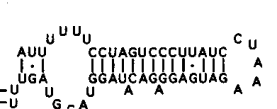

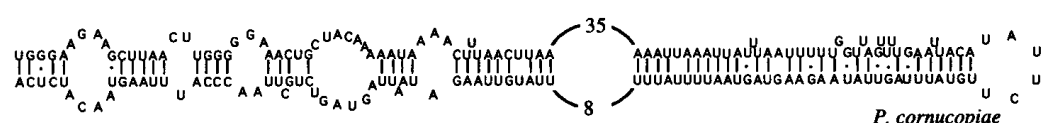

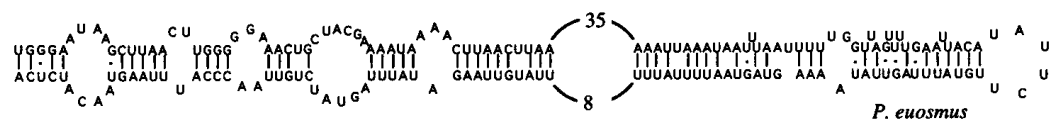

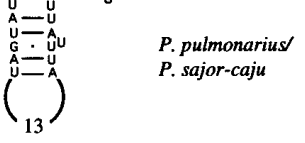

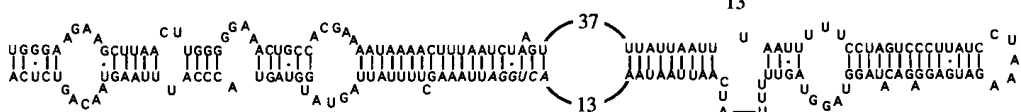
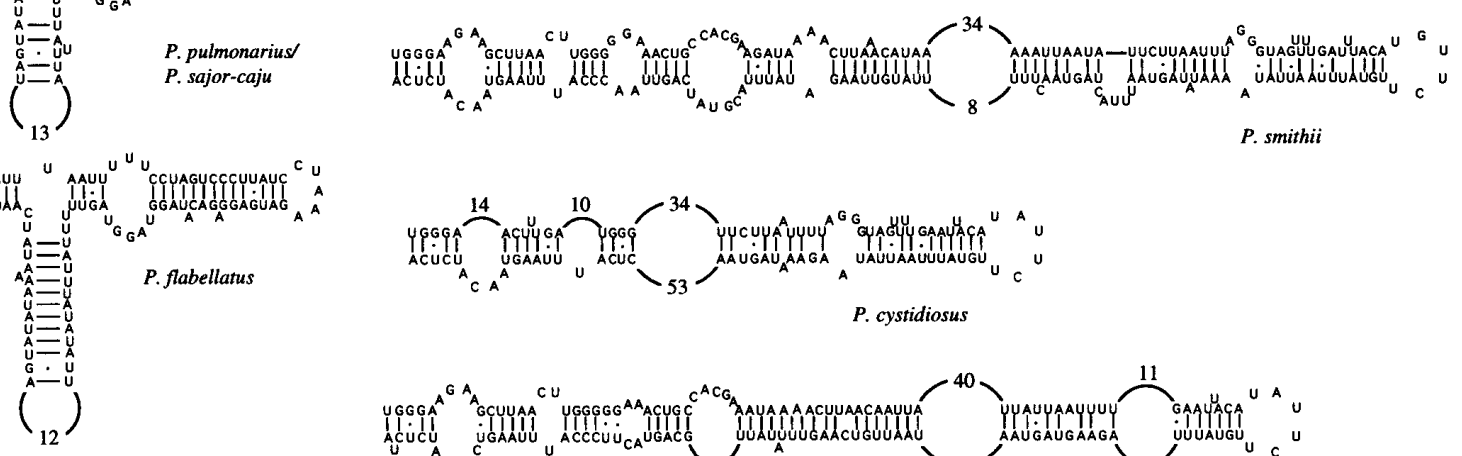

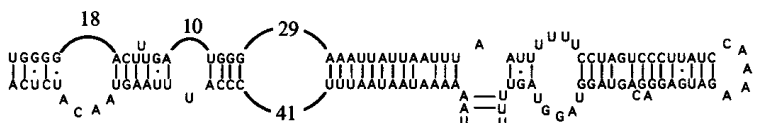

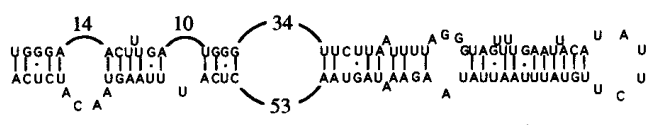

P. cystidiosus

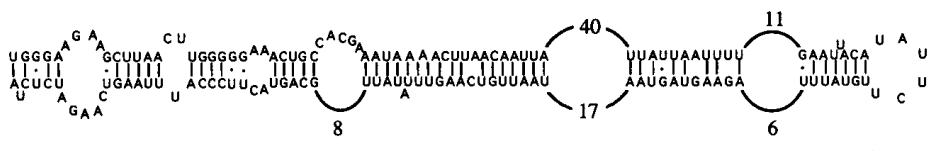

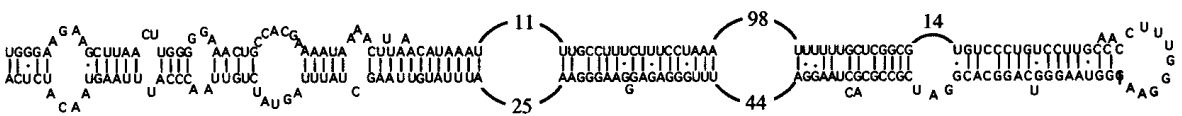

P. rattenburyi

Fig. 4. Proposed secondary structure of the variable domain V 9 of 16 species from the genus Pleurotus. The number of the additional helix is indicated. 
(a)

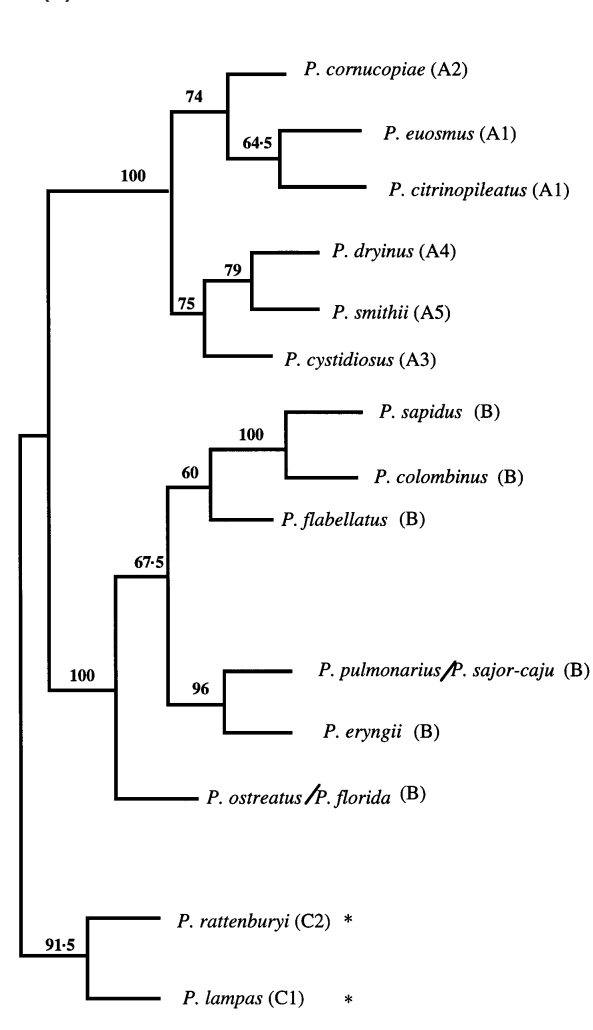

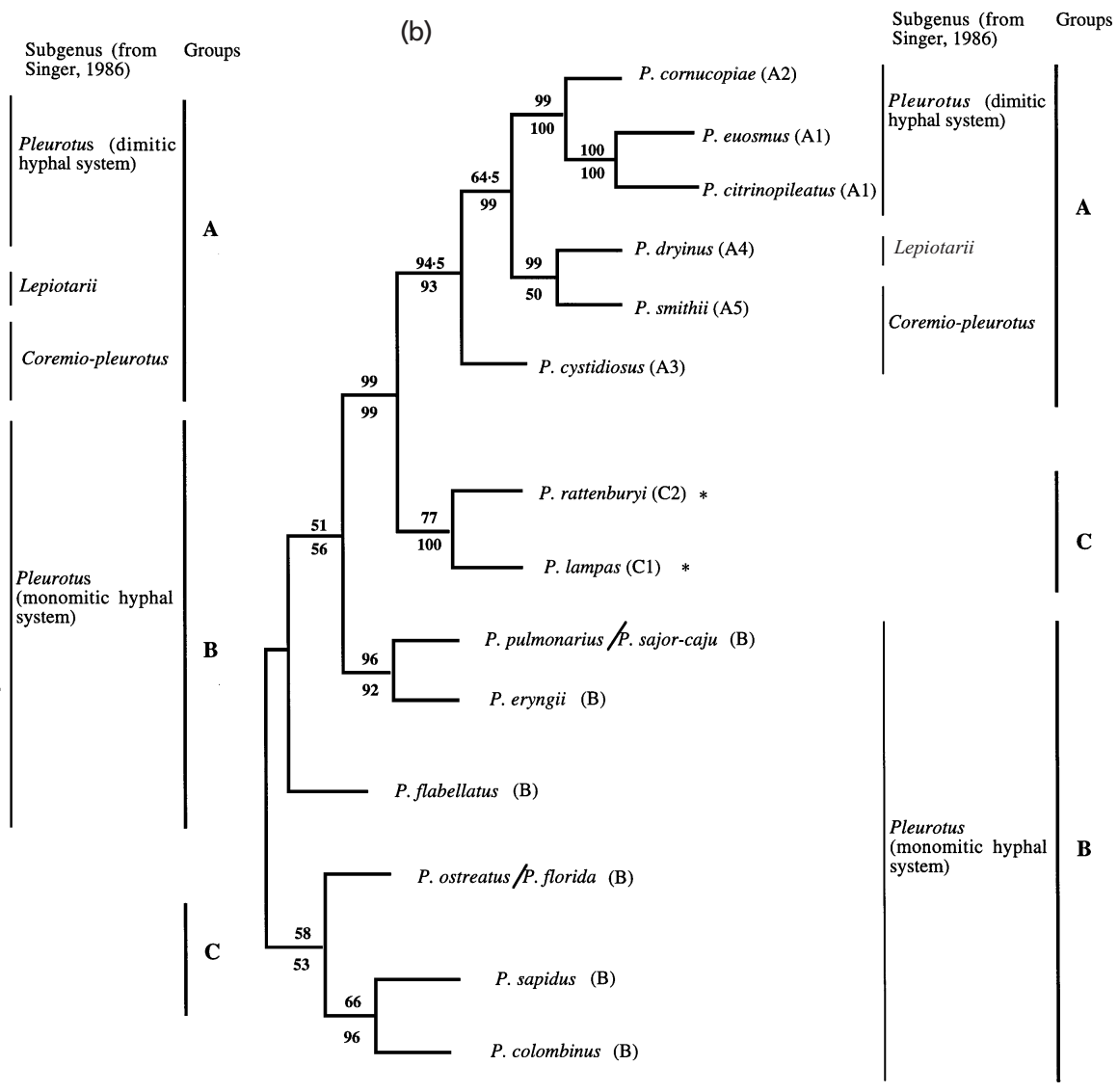

Fig. 5. (a) Consensus tree obtained by the UPGMA method. Bootstrap values greater than $50 \%$ are shown. Groups and subgroups defined by comparison of the secondary structures, and the subgenus defined by Singer (1986), are indicated. Asterisks indicate species not described by Singer. (b) Consensus tree obtained by the neighbour-joining and the parsimony methods, based upon the sequences of the variable domains V4, V6 and V9 among 16 Pleurotus species. Bootstrap values greater $50 \%$ obtained from the neighbour-joining and the parsimony methods are shown above and under branches, respectively. In each tree, subgroups are indicated.

B corresponds to species from the subgenus Pleurotus with a monomitic hyphal system, while subgroups A1 and $\mathrm{A} 2$ correspond to species from the same subgenus possessing a dimitic hyphal system. Subgroups A3 and A5 include species from the subgenus Coremiopleurotus. Species included in subgroups C1 and C2 were not described by Singer (1986).

Moreover, the three major groups (Table 1) possess structural specificities: (i) group $\mathrm{A}$ includes species without additional helices in both V4 and V9, (ii) group B includes species possessing the V4-P23-1 and the V9P49-2 additional helices, and (iii) group $\mathrm{C}$ includes species possessing a truncated V4-P23-2 helix.

\section{Relationship between Pleurotus species based on the V4, V6 and V9 sequences}

The concatenated V4-V6-V9 sequences were used to construct phylogenetic trees as described by Paquin $e t$ al. (1997). The use of such concatenated sequences gives congruent results with those obtained with separated data from V4, V6 and V9. However, the bootstrap values, and consequently the validity of these phylo- genetic trees, were higher when concatenated sequences were used.

Relationships between species within the three major groups clearly discriminated in the UPGMA phylogenetic tree, and largely recovered in the neighbourjoining/parsimony consensus tree, are in agreement with the subgenera previously described by Singer (1986) and with previous phylogenetic analysis of this genus based upon nuclear markers (Vilgalys et al., 1993; Vilgalys \& Sun, 1994; Iraçabal et al., 1995 ; Zervakis \& Balis, 1996) or isozyme markers (Zervakis et al., 1994). However, analysis of the V4, V6 and V9 domains of the mitochondrial SSU rRNA helped us to resolve some ambiguities in species designation. Indeed, sequence comparisons clearly indicated that the $P$. pulmonarius and P. sajor-caju isolates studied on the one hand, and the $P$. ostreatus and P. florida isolates studied on the other hand, shared identical sequences in their V4, V6 or V9 domains. Previous studies have shown that some $P$. pulmonarius and $P$. sajor-caju strains are interfertile (Hibber, 1982) and would probably have been misidentified (Guzman et al., 1994). Moreover, phylogenetic investigations based upon nuclear RFLP analysis were 
not able to differentiate isolates from these two species (Iraçabal et al., 1995). So, in accordance with morphological and molecular analysis, our results confirmed that the P. sajor-caju strains studied probably belong to the species $P$. pulmonarius. Similarly, the $P$. florida and $P$. ostreatus strains studied have identical nucleotide sequences. Interestingly, it has been previously suggested that strains described as $P$. florida were geographical isolates from the P. ostreatus complex (Guzman et al., 1991, 1994).

Previous phylogenetic studies have given rise to various ambiguities in the genus Pleurotus. According to the molecular markers used, P. ostreatus, $P$. colombinus and $P$. cornucopiae have been in turn associated in the same clade (Iraçabal et al., 1995) or clearly separated (Zervakis et al., 1994), and the position of P. sapidus has remained uncertain (Zervakis \& Balis, 1996). The phylogenetic analysis based upon the V4, V6 and V9 sequences reported here helps to resolve some of these ambiguities. All resulting trees show that $P$. cornucopiae on the one hand, and P. colombinus and P. ostreatus on the other hand, are clearly included in two distinct clades. It is to be noted that the close relationship between $P$. colombinus and $P$. ostreatus has been previously reported (Vilgalys et al., 1993) and that they have recently been described as sexually compatible species (Zervakis \& Balis, 1996). In another way, our results clearly demonstrate close relationships between $P$. pulmonarius and P. eryngii (96\% bootstrap support) and between P. sapidus and P. colombinus (100\% bootstrap support) (Fig. 5a), while in previous studies $P$. eryngii has been associated either with $P$. cystidiosus (Zervakis et al., 1994) or with P. ostreatus and $P$. colombinus (Iraçabal et al., 1995). Finally, the association of $P$. cornucopiae and $P$. citrinopileatus in the same clade in both consensus trees is supported by recent compatibility assays (Zervakis \& Balis, 1996).

However, the phylogenetic position of P. flabellatus remains unresolved. This species is related to $P$. sapidus and $P$. colombinus $(60 \%$ bootstrap support) by the UPGMA method and to $P$. pulmonarius/P. sajor-caju $(51 \%$ bootstrap support) by the neighbour-joining method. In an analysis based upon nuclear markers, $P$. flabellatus has been associated with $P$. cornucopiae (Vilgalys \& Sun, 1994), or considered as an intermediate between the $P$. ostreatus and the $P$. eryngii clades (Zervakis et al., 1994), or suggested to be the most divergent species of this genus, constituting an outgroup (Iraçabal et al., 1995). In the same way, P. rattenburyi and P. lampas are associated in the same clade in both consensus trees, but the phylogenetic position of this clade varied according to the method used to construct the tree.

\section{Concluding remarks}

Our results obtained from species of Pleurotus show that the V4, V6 and V9 domains of the mitochondrial SSU rRNA represent efficient molecular markers for the taxonomy of Basidiomycota. These results support previous findings in the genus Agrocybe (Gonzalez \& Labarère, 1998) and demonstrate the validity of the use of these species-specific mitochondrial sequences in the taxonomy of Basidiomycota. Moreover, nucleotide sequences of these mitochondrial domains appear to be a good alternative for defining phylogenetic relationships between species.

One of the major problems in microbiology is to identify markers strictly specific to a species. Hence, the morphological and molecular study of numerous isolates is necessary to define a species. The sequences of the V4, V6 and V9 domains of the mitochondrial SSU rRNA seem to provide such species-specific markers. The use of these sequences could have numerous applications: for example to discriminate misidentified species in culture collections, to determine the taxonomic position of unidentified species, or to give objective and unvariable criteria to determine a species. However, our studies to date are limited to two genera and future investigations would have to be extended to other genera of Basidiomycota with the aim of establishing a database for these mitochondrial molecular data.

\section{ACKNOWLEDGEMENTS}

This work was supported by grants from the Conseil Scientifique de l'Université Victor Segalen Bordeaux 2, the Conseil Régional d'Aquitaine, and the Institut National de la Recherche Agronomique. The authors thank J. L. Reigne and C. Ducos for technical help.

\section{REFERENCES}

Barroso, G. \& Labarère, J. (1995). Molecular organization and heredity of the mitochondrial genome in Basidiomycetes. Mycoscience 36, 135-142.

Barroso, G., Perennes, D. \& Labarère, J. (1995). A 'miniprep' method for RFLP analysis and dsRNAs detection perfected in the cultivated fungus A. aegerita. In Science and Cultivation of Edible Fungi, vol. 1, pp. 87-94. Edited by T. J. Elliot. Rotterdam: A. A. Balkema.

Bruns, T. D. \& Szaro, T. M. (1992). Rate and mode differences between nuclear and mitochondrial small subunit rRNA genes in mushrooms. Mol Biol Evol 9, 836-855.

Gardes, M., Fortin, A., Mueller, G. M. \& Kropp, B. R. (1990). Restriction fragment length polymorphism in the nuclear ribosomal DNA of four Laccaria spp.: L. bicolor, L. laccata, L. proxima, and L. amethystina. Phytopathology 80, 1312-1317.

Gonzalez, P. \& Labarère, J. (1998). Sequence and secondary structure of the mitochondrial small-subunit rRNA V4, V6 and V9 domains reveal highly species-specific variations within the genus Agrocybe. Appl Environ Microbiol 64, 4149-4160.

Gonzalez, P., Barroso, G. \& Labarère, J. (1997). DNA sequence and secondary structure of the mitochondrial small subunit ribosomal RNA coding region including a group-IC2 intron from the cultivated Basidiomycete Agrocybe aegerita. Gene 184, 55-63.

Guzman, G., Bandala, V. M. \& Montoya, L. (1991). A comparative study of teleomorphs and anamorphs of Pleurotus cystidiosus and Pleurotus smithii. Mycol Res 95, 1264-1269. 
Guzman, G., Montoya, L., Mata, G. \& Salmones, D. (1994). Studies in the genus Pleurotus. III. The varieties of P. ostreatus-complex based in interbreeding strains and in the study of Basidiomata obtained in culture. Mycotaxon 50, 365-378.

Hibbet, D. S. \& Donoghue, M. J. (1995). Progress toward a phylogenetic classification of the Polyporaceae through parsimony analysis of mitochondrial ribosomal DNA sequences. Can J Bot 73 (Suppl. 1), S853-S861.

Higgins, D. G. \& Sharp, P. M. (1989). Fast and sensitive multiple alignment sequence on a microcomputer. CABIOS 5, 151-153.

Hilber, O. (1982). Die Gattung Pleurotus (Fr) Kummer unter besonderer Berücksichtigung des Pleurotus eryngii-Formenkomplexes. Bibl Mycol 87, 1-448.

Iraçabal, B., Zervakis, G. \& Labarère, J. (1995). Molecular systematic of the genus Pleurotus: analysis of the restriction polymorphism in ribosomal DNA. Microbiology 141, 1479-1490.

Juan, C., Oromi, P. \& Hewitt, G. M. (1996). Phylogeny of the genus Hegeter (Tenebrionidae, Coleoptera) and its colonization of the Canary islands deduced from cytochrome oxidase I mitochondrial DNA sequence. Heredity 76, 392-403.

Khon, L. M., Petsche, D. M., Bailey , S. R., Novak, L. A. \& Anderson, J. B. (1988). Restriction fragment length polymorphism in nuclear and mitochondrial DNA of Sclerotinia species. Phytopathology 78, 1047-1051.

Molcalvo, J. M., Wang, H. H. \& Hseu, R. S. (1995). Phylogenetic relationships in Ganoderma inferred from the internal transcribed spacer and $25 \mathrm{~S}$ ribosomal DNA sequences. Mycologia 87, 223-238.

Mueller, F. \& Brimacombe, R. (1997). A new model for the threedimensional folding of Escherichia coli $16 \mathrm{~S}$ ribosomal RNA. II. The RNA-protein interaction data. J Mol Biol 271, 545-565.

Neefs, J. M., Van de Peer, Y., De Rijk, P., Chapelle, S. \& De Wachter, R. (1993). Compilation of small ribosomal subunit RNA structures. Nucleic Acids Res 21, 3025-3049.

Noller, H. F. \& Lake, J. A. (1984). Ribosome structure and function: localization of rRNA. In Membrane Structure and Function, pp. 217-297. Edited by E. Bittar. New York: Wiley.

Paquin, B., Forget, L., Roewer, I. \& Lang, B. F. (1995). Molecular phylogeny of Allomyces macrogynus: congruency between nuclear ribosomal RNA- and mitochondrial protein-based trees. $J$ Mol Evol 41, 657-665.
Paquin, B., Laforest, M. J., Forget, L., Roewer, I., Wang, Z., Longcore, J. \& Lang, B. F. (1997). The fungal mitochondrial genome project: evolution of fungal mitochondrial genomes and their gene expression. Curr Genet 31, 380-395.

Sanger, F., Nicklen, S. \& Coulson, A. R. (1977). DNA sequencing with chain-terminating inhibitors. Proc Natl Acad Sci USA 74, 5463-5467.

Singer, R. (1986). The Agaricales in Modern Taxonomy, 4th edn. Koenigstein, Germany: Koeltz Scientific Books.

Smith, M. L. \& Anderson, J. B. (1989). Restriction fragment length polymorphism in mitochondrial DNAs of Armillaria: identification of North American biological species. Mycol Res 93, 247-256.

Spicer, G. S. (1995). Phylogenetic utility of the mitochondrial cytochrome oxidase gene: molecular evolution of the Drosophila buzzatii species complex. J Mol Evol 41, 749-759.

Stern, S., Powers, T., Changchien, L. M. \& Noller, H. F. (1989). RNA-protein interactions in $30 \mathrm{~S}$ ribosomal subunit: folding and function of $16 \mathrm{~S}$ rRNA. Science 244, 783-790.

Teissier, L. H., Van der Speck, H., Gualberto, J. M. \& Grienenberger, J. M. (1997). The cox I gene from Euglena gracilis: a protist mitochondrial gene without introns and genetic code modifications. Curr Genet 31, 208-213.

Vilgalys, R. \& Sun, B. (1994). Ancient and recent patterns of geographic speciation in the oyster mushroom Pleurotus revealed by phylogenetic analysis of the ribosomal DNA sequence. Proc Natl Acad Sci USA 91, 4599-4603.

Vilgalys, R., Smith, A., Sun, B. \& Miller, O. K. (1993). Intersterility groups in the Pleurotus ostreatus complex from the continental United States and adjacent Canada. Can J Bot 71, 113-128.

Zervakis, G. \& Balis, C. (1996). A pluralistic approach in the study of Pleurotus species with emphasis on compatibility and physiology of the European morphotaxa. Mycol Res 100, 717-731.

Zervakis, G., Sourdis, J. \& Balis, C. (1994). Genetic variability and systematics of eleven Pleurotus species based on isozyme analysis. Mycol Res 98, 329-341.

Received 12 July 1999; revised 1 October 1999; accepted 12 October 1999. 